\title{
Modern GC-MS Technology in the Forensic Laboratory
}

\author{
Beat Aebi* and Werner Bernhard
}

\begin{abstract}
GC-MS is one of the most important techniques in today's forensic laboratory. It offers the possibility to screen biological and illicit drug samples for unknown compounds, to identify, confirm, and to quantitate them. This technique is very versatile and robust. The ionization technique of electron impact is standard technology; it enables the use of mass spectral libraries with hundred thousands of reference spectra. The combination of a separation technique (GC) and a spectroscopic technique (MS) results in a very dependable analytic system that is highly respected in court. The presented work will focus on some of the latest developments and will also give information on routine work.
\end{abstract}

Keywords: Forensics · GC-MS · Illicit drugs · MassLib · Toxicology

\section{Introduction}

Analytical chemistry applied to the forensic sciences is based on numerous chromatographic and spectroscopic techniques. These can be classified as screening and confirmation techniques and are applied to forensic chemistry (illicit drugs and poisons) and forensic toxicology (e.g. incapacitating consumption and traffic accidents, post-mortem toxicology). Toxicological screenings are generally described as general unknown analyses (GUA) and systematic toxicological analyses (STA). When no or only little information exists concerning the intake of illegal drugs, medications or poisons, this route is generally chosen. For both screening and identification of pharmacologically active compounds, gas chromatography combined with mass spectrometry (GC-MS) plays a key role. GC-MS offers the possibility to specifically detect thousands of compounds. This is made possible with the use of mass spectral databases. The standard-

\footnotetext{
"Correspondence: Dr. B. Aebi

University of Bern

Institute of Legal Medicine

Forensic Chemistry and Toxicology Division

Buehlstrasse 20

CH-3012 Bern

Tel.: +41316318411

Fax: +41316313833

E-Mail: beat.aebi@irm.unibe.ch

www.irm.unibe.ch
}

ized method of electron impact ionization produces generally comparable mass spectra from all commercially available GC-MS. With the possibilities of modern communication, chromatograms can be distributed electronically (e.g. E-mail) and be evaluated by various spectroscopists. If the compounds of interest are identified a confirmation step is required for forensic evidence. Finally, the identified and confirmed compounds are quantitated to evaluate their toxicological mode and degree of action. In forensic chemistry, seized illicit drugs are quantitated to obtain the total amount of the pure drug. All toxicological (or chemical) findings are compiled and compared to the other facts of the forensic case, resulting in the forensic statement and occasionally, testimony in court.

\section{Screening Techniques}

Illicit or suspected drugs are usually dissolved in an organic solvent and directly analyzed. Biological samples (e.g. blood, urine, tissue) however must be extracted first. Very reliable extraction techniques are described in the literature. These techniques are based on the distribution of compounds in aqueous and organic solvents. Using buffer solutions with defined $\mathrm{pH}$ values enables the selective transfer of compounds or classes of compounds of interest from the biological sample into an adequate organic solvent. Extractions can be specific for certain compounds or more general for basic, neutral, and acidic compounds. A thorough documentation to this technique is given by Pfleger et al. [1]. The extractions are normally performed using conventional liquid-liquid extraction (LLE) or nowadays more frequently by solid phase extraction (SPE). Semi-selective SPE cartridges are commercially available for a variety of compounds and classes of compounds. Special SPE cartridges, optimized for screening purposes, are also found on the market. Most recently, SPE units for the selective extraction of certain compounds like lysergic acid diethyl amide (LSD) have been proposed. This new type of extraction method is based on non-covalent binding of drug molecules to immobilized antibodies. After the extraction itself, chemical derivatization is frequently used to amend more polar or thermally unstable compounds to GC-MS. Most common are the formation of methyl-, trimethylsilyl-, acetyl- and trifluoroacetyl derivatives.

Fig. 1 shows a typical example of a toxicological screening analysis for basic pharmacologically active compounds in human cardiac blood, obtained after medico-legal autopsy. The upper part shows the mass spectra of the two identified compounds amitriptylin (A) and levomepromazin (B) after automatic background subtraction. The lower part shows the reconstructed ion chromato- 
gram (RIC) of the extract obtained (RI0) and of the extract after derivatization with acetic anhydride (RI 0201005d). Both chromatograms before and after chemical derivatization contain very intense signals. All but the signals of the two compounds are due to the matrix components and to chemical contaminants from the extraction procedure (mostly plasticizers like phthalates and plastic additives). In order to evaluate chromatograms efficiently, powerful search software and comprehensive mass spectral libraries are required. For several years, we have successfully been using the mass spectral search system MassLib. Additional information and demo material can be found on the Internet under www.masslib.com. MassLib was originally designed by the Max Planck Institute, Muehlheim/Ruhr, Germany, to recognize similar mass spectra of aliphatic and aromatic compounds from coal distillates. This development is in contrast to all other search programs that focus on the comparison of identical spectra. MassLib uses the search algorithm SISCOM, the search for identical and similar compounds. As can be seen in the mass spectrum of amitriptylin in Fig. 1, basic compounds in forensic chemistry and toxicology quite frequently yield mass spectra with one intense fragment only.
This is a rather large handicap for search algorithms based on the comparison of identical spectra. Another interesting feature of MassLib is the capability to search multiple libraries simultaneously [2]. Some of the libraries in current use are summarized below.

Commercial libraries for general use:

- Wiley, 7th edition, 338000 spectra, 124000 structures of 250000 compounds

- NIST 98, 12900 spectra and structures

- Steroids, 528 spectra

- Pesticides, 340 spectra

Specific commercial libraries for forensic toxicology:

- Pfleger, Maurer, Weber 3rd edition, 6300 spectra

- MassLib Tox-library, 1st edition, 2275 spectra and structures

- Finnigan Tox-library, 2200 spectra

- Designer drugs, 2nd version, 633 spectra

Non-commercial libraries for forensic toxicology:

- AAFS, 4th edition, 1833 spectra

- TIAFT, 1st version, 204 spectra
Home-build libraries for forensic toxicology:

- Unknowns IRM Berne, 184 spectra, 90 structures

- Unknowns IRM Zurich, 733 spectra (1995 version, for testing purposes)

Some of the libraries contain drawings of the chemical structures. MassLib offers the possibility to edit, save, and search for structures and sub-structures. In combination with general data searches, this proves a very helpful tool.

\subsection{Unusual Findings and Additional Tools for Identification}

Over the past three years, we have offered a service to evaluate chromatograms and spectra of other forensic institutions, namely other institutes of legal medicine and police laboratories. In most cases (total of 45), the unknown compounds were positively identified or at least, a very probable hypothesis could be proposed.

Some complex investigations involving GC-MS analyses are presented in the following case of forensic chemistry. Several suspect tablets were confiscated when an 80-year old man presented himself at the emergency desk of a local hospital with severe abdominal pain. The tablets were assumed to be designer

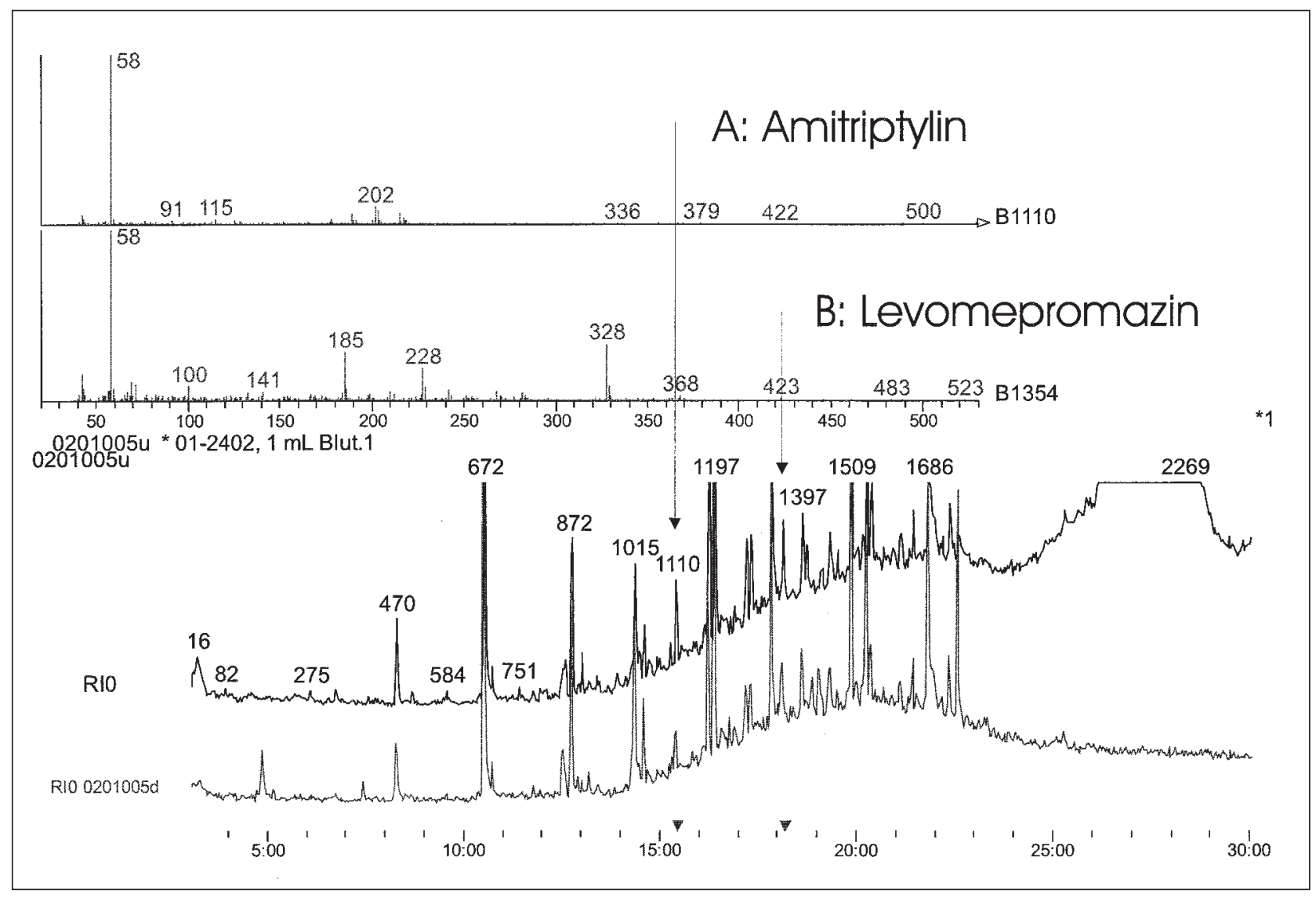

Fig. 1. Screening analysis for basic compounds. 
drugs. One of these tablets was crushed and dissolved in methanol. The corresponding chromatogram after analysis with GC-MS is shown in Fig. 2.

The spectra corresponding to compounds A8, A9, and A10 suggest a common structure of a dimethoxy-bromophenylethyl type, being similar to designer amphetamines like 2-CB and DOB. The assumption of the presence of designer drugs was hereby supported. The spectrum of A11 (and of the minor peak at no. 642) could not be identified. The comparison with all library entries suggested a substructure of the morpholinotype. Under the assumption that the tablet contained designer amphetamines, it was rather astonishing that no direct hit on a designer amphetamine was found, even with up-to-date libraries specialized on such compounds. Also the combination of hydroxy-, chlorine- and bromine-substitutions together with a morpholinosubstructure was somewhat surprising. Another possibility, namely the breakdown of another compound, probably of pharmaceutical origin, needed to be checked. With the use of the Merck Index on CD-ROM (1999 version, Hampden Data Service Ltd.), a structural search for pharmaceuticals containing a morpholino-substructure was performed. About 100 compounds were found. After visual inspection, the antispasmodic pinaverium bromide was found to contain both substructures of a dimethoxybromophenylethane and morpholinotype. The dibromo compound A10 in Fig. 2 could be explained by the degradation of pinaverium bromide and the attachment of bromine on the alkyl group. These findings were communicated to the submitting scientist. Soon after, the findings were completely confirmed by the purchase and analyses of the commercially available medication containing pinaverium bromide. Pinaverium bromide is a quaternary ammonium salt that decomposes inside the GC injector; all the compounds found are artifacts.

\subsection{GC-MS with Parallel NPD}

Most compounds of interest in forensic toxicology contain at least one nitrogen atom. This makes the use of a nitrogen-phosphorus selective detector (NPD) a very practical tool. As published before [3], the GC injector houses the capillary columns for both the MS and the NPD. By selecting the same type of capillary column but with different lengths (minus $20 \%$ for the NPD column for columns of 15 to $30 \mathrm{~m}$ ), the retention times of the signals of both detectors vary by a short time increment, typically 10 to 20 seconds. This time difference is stable and can be traced and controlled quite easily. Fig. 3 shows a typical application, again for the screening for basic compounds. Whereas as the TIC (upper trace) contains a multitude of peaks mostly of endogenous compounds, the NPD trace only manifests a few significant peaks. A rather small peak appeared at $20.53 \mathrm{~min}$ in the NPD trace. After closer examination of the TIC trace at the expected retention time of $20.24 \mathrm{~min}$, the anticonvulsant zolpidem could be identified. The chromatographic peak of zolpidem is overlapped by endogenous signals making specific spectrum subtraction inevitable. With both strong co-elution and small signal intensity, it is unlike that this compound would be found without a selective detector like the NPD, unless the target compound is already known.

\section{Confirmation Techniques}

Confirmations of chemical or toxicological findings, e.g. results from immunological or GC-MS screenings need, depending on the case, confirmation. GCMS is in most cases the method of choice to perform this task. Confirmational analyses may not only confirm a certain compound, but may also be used to differentiate individual compounds and to gain

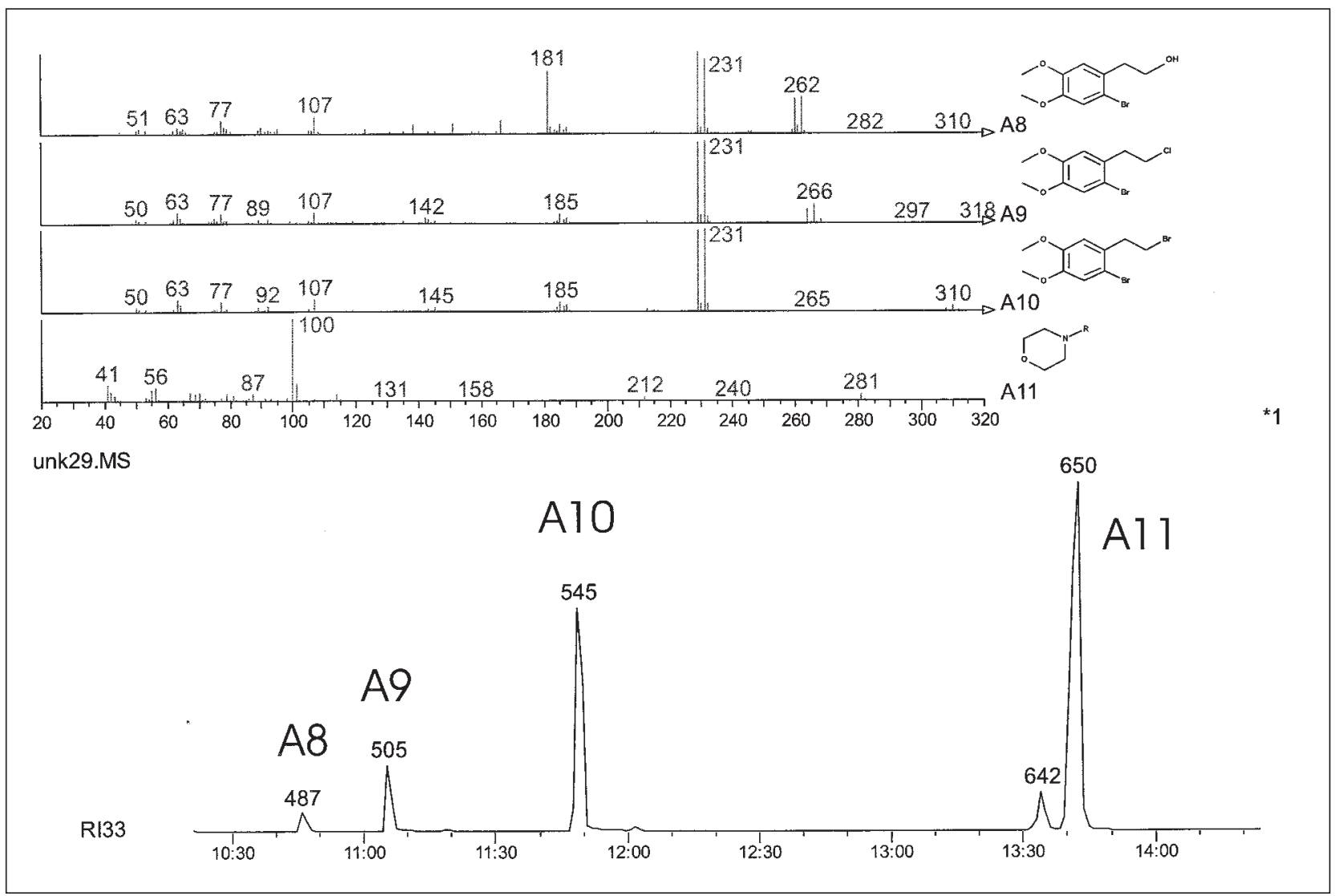

Fig. 2. Mass spectra and RIC from an unknown pill. Is it a designer drug? 
further knowledge of the case. An example is the differentiation of opiates in urine. A positive immunological test result for opiates in urine may well be an indication for an illegal consumption of heroin, but there is a variety of other possibilities. After traffic accidents, morphine is frequently administered by the emergency personnel to control pain. Codeine-containing medications are also frequently consumed. As any other opiate, codeine possesses a certain potential for drug dependency. Immunological tests are unable to differentiate between a time-limited use and a regular abuse of codeine. Cancer patients may receive morphine to limit pain. It is also widely recognized that the consumption of food containing poppy seeds may result in positive immunological tests for opiates. With the differentiation of opiates like dihydrocodeine, ethylmorphine, codeine, morphine and 6-mono-acetylmorphine (6-MAM), the source of opiates in urine can be traced. 6-MAM is the de-acetylated metabolite of heroin and proves the consumption of heroin (diacetylmorphine). After the consumption of heroin, 6-MAM can be detected for up to ten hours in the urine. The next-step metabo- lite of heroin, morphine, and codeine and their ratio allow the consumption of heroin to be traced even further back. During the illicit production of heroin, crude morphine is acetylated to form heroin. Other opiates contained in the crude morphine are acetylated in this step as well. Codeine is thereby chemically transformed to acetylcodein and again deacetylated (first step metabolism) and glucuronidated (second step metabolism) in the human body. Ratios of unconjugated morphine to codeine in urine and blood ranging from $2: 1$ to $10: 1$ indicate the consumption of illicit heroin. Heroin is also medically administered in Switzerland to treat opiate-dependent withdrawal symptoms. In contrast to illicit heroin, legally administrated heroin is chemically pure and does not contain other opiates like codeine. Whereas morphine is generally not metabolized to codeine, codeine is to a lesser extent metabolized to morphine. Many hours after the consumption of codeine, the measured levels of morphine (metabolite of codeine) may even exceed those of codeine. The interpretation of such data is even more difficult when a parallel consumption of different opiates is suspected.

\section{Quantitation Techniques}

This last step of forensic chemistry and toxicology techniques is essential to evaluate the composition of seized illegal drugs and the influence of illicit drugs and medications on the individual. Most of the toxicological cases we investigate are from driving under the influence (traffic controls and accidents). Welldocumented and thoroughly tested quantitative methods together with skilled and continuously trained personnel are required to obtain reliable results. Most quantitative methods are performed by GC-MS, partly by using isotopically marked compounds as internal standards. Due to financial reasons and availability, most standards are deuterated (e.g. morphine-D3). Labelled compounds offer several advantages over non-deuterated internal standards. Chemical properties like $\mathrm{pKa}$, solubility, and stability are very similar. Deuterated standards are very well suited to monitor and control the complete sample work-up from the extraction to the final result. Most compounds needed for routine work are commercially available as deuterated standards.

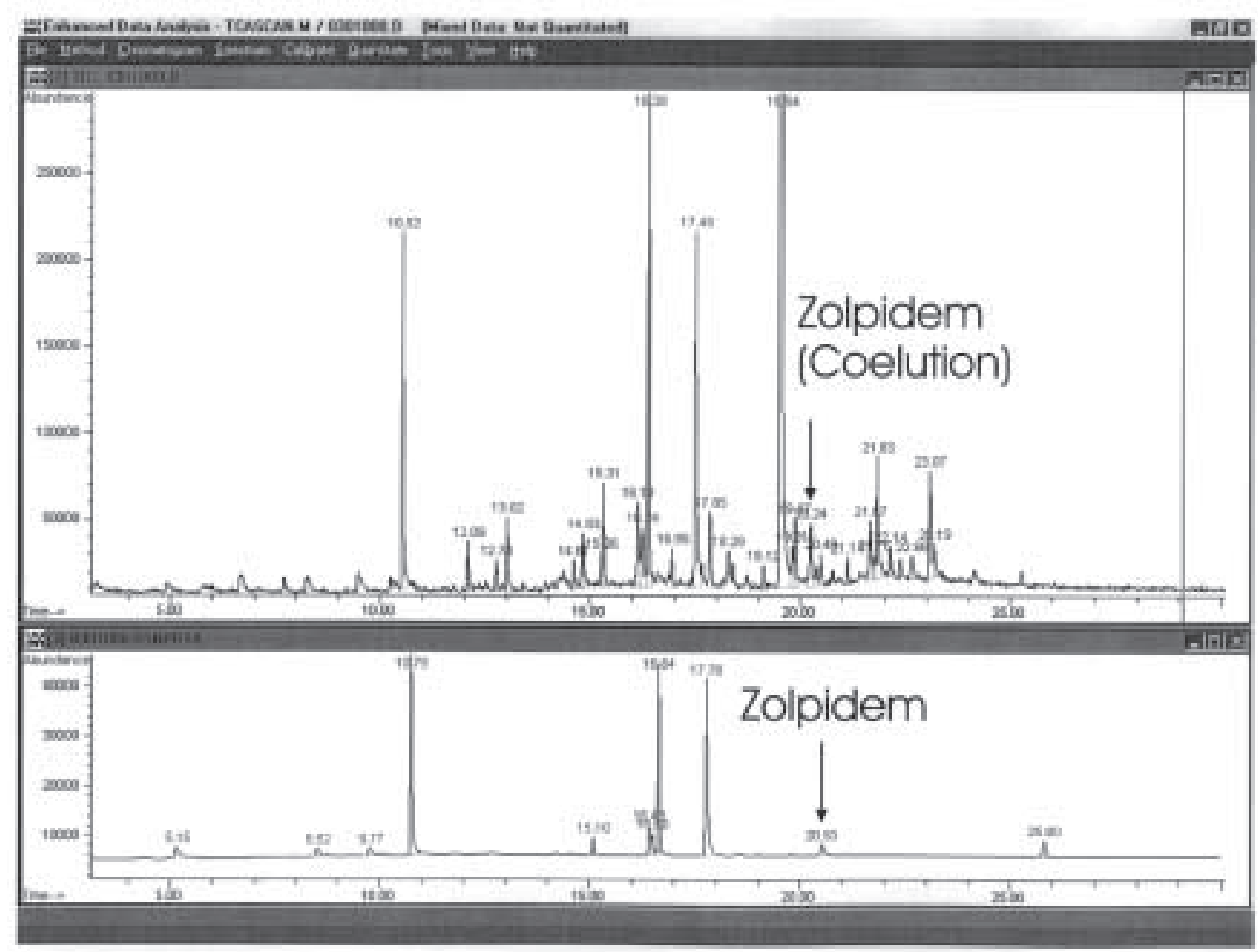

Fig. 3. GC-MS analysis with parallel NPD 


\section{GC-MS and Other Techniques}

Of course, not all pharmaceutically active compounds are suited for GC-MS. Unfavorable properties like thermal instability and high polarity may exclude the use of this technique. Some of these compounds may be chemically derivatized to allow GC-MS analyses. If this is not successful, other analytical techniques may be used. Quite often, the complementary technique of liquid chromatography with photometric detection (HPLC-UV or DAD) is utilized. This technique can be further enhanced with derivatization techniques to increase the photometric sensitivity of certain compounds. Nevertheless, HPLC-UV is often not sufficiently sensitive for toxicological analyses. The combination of HPLC and MS, based on atmospheric pressure ionization interfaces (API), has become a very interesting tool in recent years. HPLC offers a very large spectrum of possibilities and MS delivers the necessary sensitivity. For the quantitation of a large variety of compounds, including thermally instable and highly polar ones, LC-MS is the method of choice. But LCMS has not (yet) the power of GC-MS with regards to separation power and identification of unknown compounds. Several groups at universities and analytical instrument companies are currently working on the introduction of LC-MS spectral databases for screening purposes [4][5]. Unlike the standardized electron impact ionization of GC-MS, LC-MS depends on several parameters defining the formation of ions. Buffers, pHs, pKas, solubility, liquid flows, ionization voltages, declustering and fragmentation voltages as well as interface temperature are parameters that can have a pronounced effect on the production and detectability of ions from the liquid phase. Moreover, solvents and dissolved compounds tend to form clusters and artifacts. Up to now, these inconveniences prevented the introduction of a systemindependent method for screening analyses. Besides the standardization of LCMS techniques, more efforts are made using MS/MS techniques and online data evaluation processes. The data system decides if a chromatographic peak is significant and programs itself the necessary MS/MS scan. The recognition of interesting peaks as well as the processing and acquisition of MS/MS data happens almost simultaneously. A chromatographic peak can be analyzed by MS and MS/MS at almost the same time.
Further progress was achieved with GC-MS. Whereas the application of MS/ MS techniques has been established for many years, the use of time-of-flight technology (TOF) has only just begun. Ion detection of TOF instruments is very rapid and these instruments also offer a higher ion transmission. In contrast to conventional MS analyzers and detectors (quadrupoles, ion traps, sector instruments), where ions are separated and detected one at a time using a mass scan, TOF instrument acquire the whole spectrum in a fast manner. TOF instruments are not only capable of obtaining several thousand spectra per second, but can also to do this at a high resolution. Similar to other well-known high-resolution techniques (e.g. sector instruments), the data system calculates the most probable elemental composition of a mass peak of interest based on the exact mass of the involved isotopes. GC-TOF in combination with EI yields mass spectra that are well suited for searching in standard GCMS spectral libraries [6].

\section{Experimental}

Screening analysis for basic pharmacologically active compounds in human blood (section 2): $1 \mathrm{ml}$ of whole blood was spiked with the internal standard proadifen, brought to $\mathrm{pH} 9$ with buffer solution and vortexed. After the addition $2 \mathrm{ml}$ of ethyl acetate, the sample was vortexed again. The supernatant was transferred and the solvent evaporated. The dry residue was reconstituted with $50 \mu \mathrm{l}$ of ethyl acetate. Of this solute, $2 \mu l$ were injected into the GC-MS.

GC-MS-NPD system 1: GC 5890 Series II, MSD 5972 with NPD and 6890 autosampler (all Agilent Technologies), two separation columns connected inside the standard split/splitless injector using a two-bore ferrule. Capillary columns from J\&W (Agilent), DB5-ms, ID 0.25 $\mathrm{mm}$, stationary phase $0.25 \mu \mathrm{m}$ of $13 \mathrm{~m}$ length for the NPD and $17 \mathrm{~m}$ for the MSD.

GC-MS-NPD/TSD system 2: SSQ 7000 mass spectrometer (Thermo Finnigan) with a CX3400 gas chromatograph (Varian), equipped with an Agilent standard split/splitless injector, an automated liquid sampler A200S (CTC Analytics) and a thermoionic detector TSD (Varian), specific for nitrogen- and phosphorus-containing compounds. Both separation columns were connected inside the injector using a two-bore ferrule. Separation columns from J\&W, DB5-ms,
$0.25 \mathrm{~mm}$ ID, stationary phase $0.25 \mu \mathrm{m}$. Lengths of the capillaries: $30 \mathrm{~m}$ for the MS, $25 \mathrm{~m}$ for the TSD.

\section{Summary}

GC-MS techniques are currently the gold standard for the identification, confirmation, and quantitation of compounds in the field of forensic chemistry and toxicology. The knowledge of extraction and derivatization techniques is quite vast. On the other hand, new compounds of higher complexity and also higher pharmacological potency are being developed at a fast pace. Very promising advances for GC-MS are currently proposed by the use of time-of-flight mass spectrometers coupled to gas chromatography (TOF-GS-MS). Future development of analytical methods certainly will be directed towards LC-MS and LC-MS-MS. A big step to further extend the use of LC-MS in forensic chemistry and toxicology will be the possibility of searching for unknown compounds, currently limited by the number of available reference spectra containing sufficient fragment information.

Received: January 22, 2002

[1] K. Pfleger, H.H. Maurer, A. Weber, 'Mass Spectral and GC Data of Drugs, Poisons, Pesticides, Pollutants and their Metabolites', 2nd ed., part 4, Wiley-VCH, D-Weinheim, 2000.

[2] B. Aebi, W. Bernhard, 'Advances in the Use of Mass Spectral Libraries for Forensic Toxicology', J. Analyt. Toxicol. 2002, in press.

[3] B. Aebi, W. Bernhard, 'Gas chromatography with dual mass spectrometric and nitrogen-phosphorus specific detection: A new and powerful tool for forensic analyses', For. Sci. Int. 1999, 102, 91-101.

[4] W. Weinmann, A. Wiedemann, B. Eppinger, M. Renz, 'Screening for Drugs in Serum by Electrospray Ionization/Collision-Induced-Dissociation and Library Searching', J. Am. Soc. Mass Spectrom. 1999, 10, 1028-1037.

[5] P. Marquet, N. Venisse, E. Lacassie, G. Lachâtre, 'In-source CID mass spectral libraries for the 'general unknown' screening of drugs and toxicants', Analusis 2001, 28(10), 925-934.

[6] B. Aebi, R. Sturny-Jungo, W. Bernhard, R. Blanke, R. Hirsch, 'Quantitation using GCTOF-MS: Example of Bromazepam', For. Sci. Int. 2002, special issue on the 39th TIAFT-Meeting, Prague 2001, in press. 